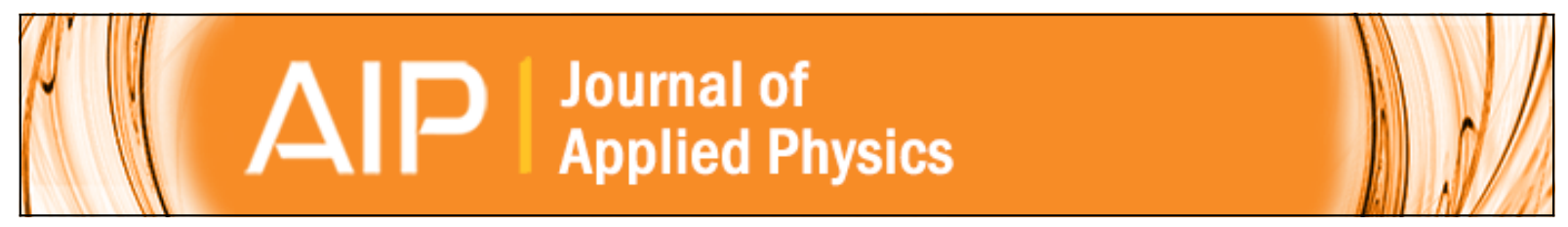

\title{
Effects of an in-plane axisymmetric magnetic field on the vibration of a thin conductive spinning disk
}

Arthur J. Michalek, Piergiovanni Marzocca, John Moosbrugger, and Davresh Hasanyan

Citation: Journal of Applied Physics 97, 10 R509 (2005); doi: 10.1063/1.1855463

View online: http://dx.doi.org/10.1063/1.1855463

View Table of Contents: http://scitation.aip.org/content/aip/journal/jap/97/10?ver=pdfcov

Published by the AIP Publishing

\section{Articles you may be interested in}

Phase-field simulation of electric-field-induced in-plane magnetic domain switching in magnetic/ferroelectric layered heterostructures

J. Appl. Phys. 109, 123917 (2011); 10.1063/1.3600203

A method for applying a large persistent in-plane biaxial stress to influence the perpendicular magnetic anisotropy of magnetic thin films

J. Appl. Phys. 102, 113910 (2007); 10.1063/1.2821309

Study of head takeoff phenomenon influenced by stick-slip vibration of slider on head-disk interface

J. Appl. Phys. 99, 08N104 (2006); 10.1063/1.2172533

Multipole moments of in-plane magnetized disks

J. Appl. Phys. 97, 10J502 (2005); 10.1063/1.1847351

A cell for in-plane, isotropic external stressing of thin films: Inverse magnetoelastic effects

Rev. Sci. Instrum. 68, 1796 (1997); 10.1063/1.1147995

\section{MIT LINCOLN} LABORATORY CAREERS

Discover the satisfaction of innovation and service to the nation
- Space Control

- Air \& Missile Defense

- Communications Systems \& Cyber Security

- Intelligence, Surveillance and

Reconnaissance Systems
- Advanced
Electronics
- Tactical Systems
- Homeland
Protection
- Air Traffic Control

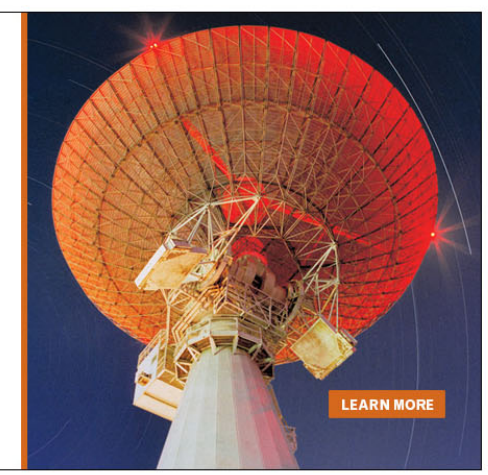




\title{
Effects of an in-plane axisymmetric magnetic field on the vibration of a thin conductive spinning disk
}

\author{
Arthur J. Michalek, Piergiovanni Marzocca, and John Moosbrugger \\ Department of Mechanical and Aeronautical Engineering, Clarkson University, Potsdam, New York 13699
}

Davresh Hasanyan

Department of Engineering Science and Mechanics, Virginia Tech, Blacksburg, Virginia 24061

(Presented on 9 November 2004; published online 17 May 2005)

\begin{abstract}
This paper details the derivation and solution of the governing equation for linear transverse vibrations of a thin perfectly conducting rotating disk subjected to an axisymmetric in-plane magnetic field having a circumferential flux pattern. Previous works on plates and shells have hypothesized that the application of a magnetic field is capable of increasing the natural frequency of a thin conductive plate. Analytical results show a significant increase in maximum stable operating speed of a thin disk in the presence of a magnetic field. The effect is dependent on both thickness and magnetic field strength. (C) 2005 American Institute of Physics.
\end{abstract}

[DOI: 10.1063/1.1855463]

\section{INTRODUCTION}

Thin, spinning disks have a broad range of mechanical applications, including data storage drives, circular saws, and turbomachinery. In these applications, it is desirable to have the thinnest possible disk spinning at the highest possible angular velocity. It is also desirable to keep transverse vibrations to a minimum. ${ }^{1}$ In a computer disk drive, for example, transverse vibration can have consequences ranging from track misregistration ${ }^{2}$ to a catastrophic failure. It is thus important to avoid exciting resonance frequencies during the operation of these devices. In a stationary disk, there are a series of vibrational modes consisting of waves traveling in opposite directions around the disk, with the natural frequencies of the two waves of each mode being the same. As the disk is spinning, the frequencies of the waves traveling in the direction of the angular displacement increase, while the frequencies of the waves traveling counter to the angular displacement decrease. As angular velocity is increased, the frequencies of the backward traveling waves diminish to zero. The lowest velocity at which any of the modes has a zero frequency is referred to as the critical speed. After this point, the disk is considered to be elastically unstable. At higher speeds, aerodynamic coupling effects ${ }^{3}$ cause the disk to flutter. Recent developments in the field ${ }^{4}$ have pointed toward active control models to reduce these vibrations.

A method to passively increase the critical speed of a thin conductive spinning disk using an electromagnetic field is presented in this paper. The magnetoelastic governing equation accounting for the electromagnetic forces generated by an electric field coupled with the elasticity of the thin disk is analytically derived. The application of an in-plane electromagnetic field on a thin rectangular strip of a conductive material has been shown ${ }^{5}$ to produce an increase in stiffness and consequently in the natural frequency. In this paper it is shown that the application of an axisymmetric, in-plane electromagnetic field produces an increase in the critical speed of a spinning disk. The field in this case is that would result from a current carrier concentric with the disk and oriented perpendicularly to its surface.

\section{DEVELOPMENT OF GOVERNING EQUATIONS}

The magnetoelastic governing equations of spinning disks are derived upon the following assumptions: (i) the disk is modeled as Kirchhoff's plate, thus there is no variation in the rotational stresses throughout the thickness of the plate; (ii) the vibrations in the disk are assumed to be linear, and bending stresses are negligible; (iii) the disk is secured by a rigid clamp, which allows no displacement at the inner radius; (iv) there is no slipping between the clamp and the disk; (v) the effect of rotary inertia is considered to be negligible; and (vi) as it only effects vibrational modes that do not have critical speeds, ${ }^{6}$ disk-spindle coupling is neglected.

The resulting mechanical model has been widely used ${ }^{7}$ and shown to closely follow predict findings.

Assuming that the disk is immersed in a magnetic field, which is axisymmetric and constant in time, and spinning at a constant angular velocity $\Omega$ the governing equation of transverse vibration, in a fixed system of coordinates may be expressed as follows:

$$
\begin{aligned}
\frac{\partial^{2} w}{\partial t^{2}} & +2 \Omega \frac{\partial^{2} w}{\partial \theta \partial t}+\Omega^{2} \frac{\partial^{2} w}{\partial \theta^{2}}+\nabla^{4} w-\frac{1}{r} \frac{\partial}{\partial r}\left(r \sigma_{r} \frac{\partial w}{\partial r}\right) \\
& -\frac{\sigma_{\theta}}{r^{2}} \frac{\partial^{2} w}{\partial \theta^{2}}-q(r, \theta, t)=0,
\end{aligned}
$$

where $w(r, \theta, t)$ denotes the transverse deflection of the middle plane of the disk, $\sigma_{r}$ and $\sigma_{\theta}$ are the in-plane rotational stresses and are defined using the zero displacement model, ${ }^{6}$ $q(r, \theta, t)$ is the transverse load resulting from the magnetic field, and assuming a perfectly conductive disk, may be expressed as follows: 


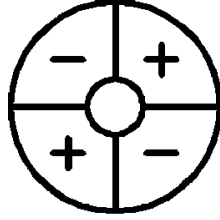

(0,2)

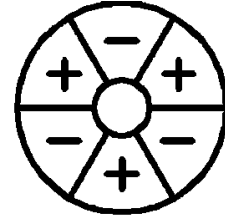

$(0,3)$

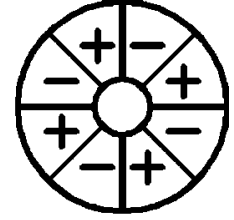

$(0,4)$
FIG. 1. Definition of modal shapes.

$$
\begin{aligned}
q(r, \theta, t)= & -H_{0 \theta}\left[h_{\theta}^{(e)+}-h_{\theta}^{(e)-}\right]+H_{0 \theta}\left[h_{\theta}^{+}-h_{\theta}^{-}\right] \\
& -H_{0 r}\left[h_{r}^{(e)+}-h_{r}^{(e)-}\right]+H_{0 r}\left[h_{r}^{+}-h_{r}^{-}\right] \\
& +\int_{-h}^{h}\left\{f_{3}+z\left[\cos \theta \frac{\partial}{\partial r}-\sin \theta \frac{\partial}{\partial \theta}\right] f_{1}\right. \\
& \left.+z\left[\sin \theta \frac{\partial}{\partial r}+\cos \theta \frac{\partial}{\partial \theta}\right] f_{2}\right\} d z,
\end{aligned}
$$

where $\vec{h}^{(e)}\left(h_{r}^{(e)}, h_{\theta}^{(e)}\right)$ and $\vec{h}\left(h_{r}, h_{\theta}\right)$ are disturbed quantities of magnetic field, $\vec{H}_{0}\left(H_{0 r}, H_{0 \theta}\right)$, generated by the deformation of the plate outside and inside of the disk area, respectively.

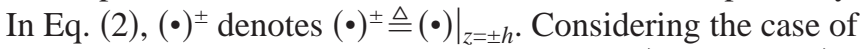
a current carrier concentric with the disk $\vec{H}_{0 r}=0$ and $\vec{H}_{0 \theta}$ $=\left(J_{0} / 2 \pi r\right) \vec{e}_{\theta}=H_{0} \vec{e}_{\theta}$. Herein $J_{0}$ is the current density.

The components of the perturbed magnetic field inside the perfectly conductive disk are determined ${ }^{7}$ by $\vec{h}=\operatorname{rot}(\vec{u}$ $\left.\times \vec{H}_{0}\right)$. The body forces $\vec{f}$ can be expressed in the form $\vec{f}$ $=\operatorname{rot} \vec{h} \times \vec{H}_{0}$. The magnetic field, $h_{r}^{(e)}, h_{\theta}^{(e)}, h_{z}^{(e)}$, outside the disk area are determined by solving the following boundary problem:

$$
\nabla^{2} \phi^{(e)}=0 ; \frac{\partial \phi^{(e)}}{\partial z}=\left.h_{z}\right|_{z= \pm h} ; \quad \vec{h}^{(e)}=\nabla \phi^{(e)} .
$$

Assuming $w(r, \theta, t)=u(r, t) e^{i n \theta}$, with $n$ defining the number of nodal diameters, the solution of Eq. (3) may be represented in the form

$$
\begin{aligned}
\phi^{(e)}(r, \theta, z)= & e^{i n \theta} \int_{0}^{\infty} s \tilde{h}_{z}(s, \theta) d s \\
& \times \int_{0}^{\infty} e^{(h-|r|) \lambda} J_{m}(\lambda r) J_{m}(\lambda s) d \lambda d s,
\end{aligned}
$$

where $J_{m}$ is a Bessel function of the first kind of order $m$ and

$$
\tilde{h}_{z}= \begin{cases}0,0<r<a, & b<r<\infty \\ \frac{J_{0}}{2 \pi r^{2}} \frac{\partial w}{\partial \theta}, & a \leqslant r \leqslant b\end{cases}
$$

Substituting Eqs. (3) and (4) into Eq. (2), and accounting for Eq. (5), the equation of motion (1) can be converted to

$$
\begin{aligned}
\frac{\partial^{2} w}{\partial t^{2}}+ & 2 \Omega \frac{\partial^{2} w}{\partial \theta \partial t}+\Omega^{2} \frac{\partial^{2} w}{\partial \theta^{2}}+\nabla^{4} w-\frac{1}{r} \frac{\partial}{\partial r}\left(r \sigma_{r} \frac{\partial w}{\partial r}\right)-\frac{\sigma_{\theta}}{r^{2}} \frac{\partial^{2} w}{\partial \theta^{2}} \\
= & 3\left(1-\nu^{2}\right)\left(\frac{a}{h}\right)^{3} \frac{H_{0}^{2}}{E} \frac{1}{r} \\
& \times\left\{\int_{\kappa}^{1} K(r, s) \frac{1}{s} \frac{\partial^{2} w(s, \theta)}{\partial \theta^{2}} d s+\frac{h}{a} \frac{1}{r^{3}} \frac{\partial^{2} w}{\partial \theta^{2}}\right\},
\end{aligned}
$$

where

$$
K(r, s)=\int_{0}^{\infty} J_{m}(\lambda r) J_{m}(\lambda s) d \lambda .
$$

\section{SOLUTION METHODOLOGY}

The transverse displacement of the disk is approximated by the following Frobenius polynomial:

$$
w(r, \theta, t)=\left(1+A_{0} r+A_{1} r^{2}+A_{2} r^{3}+A_{3} r^{4}\right) e^{i(n \theta+\omega t)} .
$$

The constants $A_{i}$ are specified to satisfy the four boundary conditions, which define a clamped inner radius,

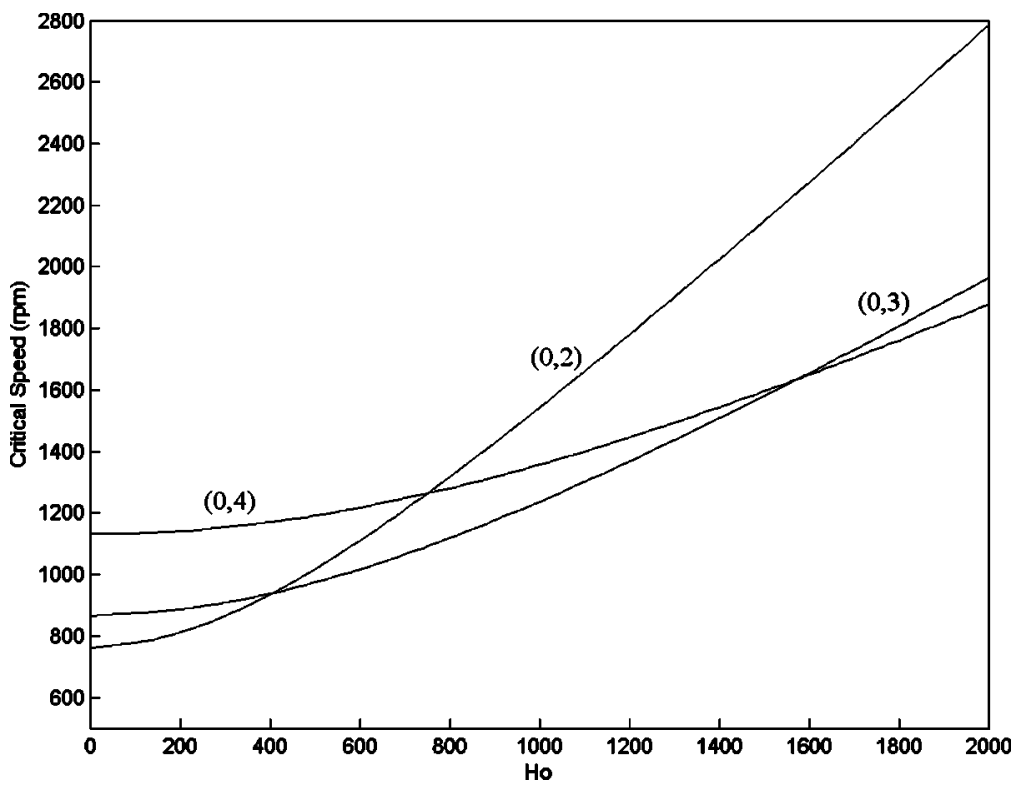

FIG. 2. Critical speeds of selected modes magnetic field intensity. 


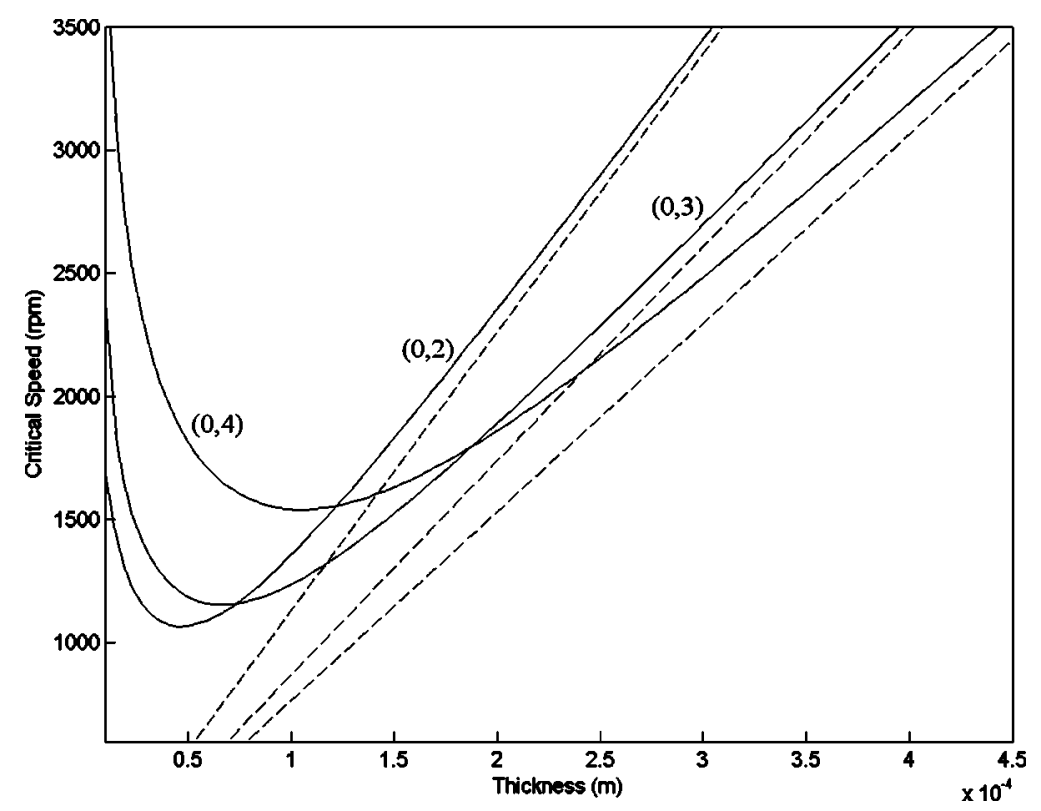

FIG. 3. Critical speeds of selected modes vs disk thickness.

$$
[w]_{r=\kappa}=0 ; \quad\left[\frac{\partial w}{\partial r}\right]_{r=\kappa}=0
$$

and a free outer radius,

$$
\begin{aligned}
& {\left[r \frac{\partial^{2} w}{\partial r^{2}}+\nu\left(\frac{\partial w}{\partial r}+\frac{1}{r} \frac{\partial^{2} w}{\partial \theta^{2}}\right)\right]_{r=1}=0 ;} \\
& {\left[r \frac{\partial}{\partial r}\left(\nabla^{2} w\right)+\frac{1-\nu}{r} \frac{\partial^{2}}{\partial \theta^{2}}\left(\frac{\partial w}{\partial r}-\frac{w}{r}\right)\right]_{r=1}=0 .}
\end{aligned}
$$

The integral in the right-hand side of Eq. (6) has been evaluated numerically, and expressed in a form consistent with Eq. (8). Replacement of Eq. (8) into the governing equation yields a residual term $L$ that is minimized in the Galerkin sense,

$$
\int_{\kappa}^{1} \mathrm{~L}[w(r, \theta, t)] w(r, \theta, t) d r=0 .
$$

Solution of Eq. (11) for $\omega$ results in a pair of roots representing the natural frequencies of the forward and backward traveling waves of the $(0, n)$ mode. Examples of these modal shapes are outlined in Fig. 1.

\section{RESULTS AND DISCUSSION}

Unless otherwise stated, the numerical results presented here consider a steel disk with a clamped radius of $25 \mathrm{~mm}$, an outer radius of $100 \mathrm{~mm}$, and a thickness of $0.1 \mathrm{~mm}$. In Fig. 2, the effect of the magnetic field strength on critical speed is plotted. It is also evident that as the strength of the magnetic field is increased, the first mode to become critical changes, and in some cases, two modes may become critical at the same speed.

The thickness of the disk has been found to have a significant interaction with the magnetic field. Figure 3 high- lights the effect of the disk thickness on the critical speed. The solid lines represent the stability loci for the first three critical modes with a magnetic field intensity of 1000 . The dashed lines indicate the stability of the disk without a magnetic field.

\section{CONCLUSIONS}

In this paper the theoretical modeling and numerical solution of the transverse vibration and critical speed of a spinning disk immersed in a magnetic field and the study of the magnetoelastic interactions are presented. Results reveal that the application of a magnetic field causes a significant increase in both the disk's stationary natural frequency and its critical speed. The effects of the magnetic field on the disk's critical speed are inversely proportional to thickness and outside radius. For practical ranges of disk geometry and field intensity, the critical speed may be increased by over $50 \%$. In applications where the operating speed of the disk is fixed, an induced magnetic field could allow the use of a disk that is thinner than would otherwise be practical. In addition, it has been found that the first vibrational mode to become unstable varies with disk geometry and magnetic field intensity.

${ }^{1}$ H. Lamb and R. V. Southwell, Proc. R. Soc. London, Ser. A 99, 272 (1921).

${ }^{2}$ A. A. Renshaw, J. Appl. Mech. 65, 166 (1998).

${ }^{3}$ S.-Y. Lee, J.-D. Kim, and S. Kim, Microsystem Technologies 9, 206 (2002).

${ }^{4}$ J.-S. Chen, Int. J. Mech. Sci. 45, 1269 (2003).

${ }^{5}$ L. Librescu, D. Hasanyan, and D. R. Ambur, Int. J. Non-Linear Mech. 39, 723 (2004)

${ }^{6}$ D. R. Chivens and H. D. Nelson, 1975, The natural Frequencies and Critical Speeds of a Rotating, Flexible Shaft-Disk System," ASME J. Eng. Ind. 97, 881 (1975).

${ }^{7}$ C. D'Angelo III and C. D. Mote, Jr., J. Sound Vib. 168, 1 (1993).

${ }^{8}$ Z. Qin, L. Librescu, D. Hasanyan, and D. R. Ambur, Int. J. Eng. Sci. 41, 2005 (2003). 